\title{
Long-term effect of rosiglitazone and/or ramipril on the incidence of diabetes
}

\author{
DREAM On (Diabetes Reduction Assessment \\ with Ramipril and Rosiglitazone Medication \\ Ongoing Follow-up) Investigators
}

Received: 23 July 2010 / Accepted: 22 October 2010 / Published online: 30 November 2010

(C) Springer-Verlag 2010

\begin{abstract}
Aims/hypothesis The Diabetes Reduction Assessment with Ramipril and Rosiglitazone Medication (DREAM) trial reported that 3 years of therapy with rosiglitazone reduced the primary outcome of diabetes or death by $60 \%$. Here we investigated whether an effect on diabetes prevention persists more than 1.5 years after therapy has been discontinued. Methods The DREAM On passive follow-up study was conducted at 49 of the 191 DREAM sites. Consenting
\end{abstract}

DREAM On Writing Group: H. C. Gerstein (McMaster University and Hamilton Health Sciences and Population Health Research Institute, Hamilton, ON, Canada), V. Mohan (Dr Mohan's Diabetes Specialities Centre, Chennai, India), A. Avezum (Research Division, Dante Pazzanese Institute of Cardiology, São Paulo, Brazil), R. M. Bergenstal (International Diabetes Center at Park Nicollet, Minneapolis, MN, USA), J.-L. Chiasson (CHUM, University of Montreal, Montreal, QC, Canada), M. Garrido (Clínica Privada Provincial, Buenos Aires, Argentina), I. MacKinnon (Instituto de Investigaciones Clinicas, Mar del Plata, Argentina), P. V. Rao (Nizam's Institute of Medical Sciences University, Hyderabad, India), B. Zinman (Samuel Lunenfeld Research Institute, Mount Sinai Hospital, University of Toronto, Toronto, ON, Canada), H. Jung (Population Health Research Institute, Hamilton, ON, Canada), L. Joldersma (Population Health Research Institute, Hamilton, ON, Canada), J. Bosch (McMaster University and Hamilton Health Sciences and Population Health Research Institute, Hamilton, ON, Canada), S. Yusuf (McMaster University and Hamilton Health Sciences and Population Health Research Institute, Hamilton, ON, Canada)

Electronic supplementary material The online version of this article (doi:10.1007/s00125-010-1985-4) contains a complete list of DREAM On Investigators, and is available to authorised users.

DREAM On (Diabetes Reduction Assessment with Ramipril and Rosiglitazone Medication Ongoing Follow-up) Investigators DREAM Project Office,

Population Health Research Institute, DBCVSRI,

237 Barton Street,

Hamilton, ON, Canada L8L 2X2

e-mail: dream@phri.ca participants were invited to have a repeat OGTT 1-2 years after active therapy ended. A diagnosis of diabetes at that time was based on either a fasting or $2 \mathrm{~h}$ plasma glucose level of $\geq 7.0 \mathrm{mmol} / 1$ or $\geq 11.1 \mathrm{mmol} / \mathrm{l}$, respectively, or a confirmed diagnosis by a non-study physician. Regression to normoglycaemia was defined as a fasting and $2 \mathrm{~h}$ plasma glucose level of $<6.1 \mathrm{mmol} / 1$ and $<7.8 \mathrm{mmol} / 1$, respectively. Results After a median of 1.6 years after the end of the trial and 4.3 years after randomisation, rosiglitazone participants had a $39 \%$ lower incidence of the primary outcome (hazard ratio [HR] $0.61,95 \%$ CI $0.53-0.70 ; p<0.0001)$ and $17 \%$ more regression to normoglycaemia $(95 \%$ CI $1.01-1.34$; $p=0.034)$. When the analysis was restricted to the passive follow-up period, a similar incidence of both the primary outcome and regression was observed in people from both treatment groups (HR 1.00, 95\% CI $0.81-1.24$ and HR $1.14,95 \%$ CI $0.97-1.32$, respectively). Similar effects were noted when new diabetes was analysed separately from death. Ramipril did not have any significant long-term effect.

Conclusions/interpretation Time-limited exposure to rosiglitazone reduces the longer term incidence of diabetes by delaying but not reversing the underlying disease process.

Keywords Diabetes prevention · Legacy effect - Ramipril · Randomised $\cdot$ Rosiglitazone

$\begin{array}{ll}\text { Abbreviations } \\ \text { DREAM } & \begin{array}{l}\text { Diabetes Reduction Assessment with } \\ \text { Rosiglitazone and Ramipril Medication }\end{array} \\ \text { FPG } & \text { Fasting plasma glucose } \\ \text { IFG } & \text { Impaired fasting glucose } \\ \text { IGT } & \text { Impaired glucose tolerance } \\ \text { PG } & \text { Plasma glucose }\end{array}$




\section{Introduction}

Diabetes is a common chronic condition that is rapidly increasing in prevalence. Approximately $6.5 \%$ of adults globally have diabetes and type 2 diabetes accounts for more than $90 \%$ of cases; in addition, $8 \%$ have impaired glucose tolerance (IGT) [1]. In the past 9 years, several large randomised trials conducted throughout the world have clearly demonstrated that lifestyle interventions comprising modest changes in diet and/or physical activity can dramatically reduce the incidence of type 2 diabetes in high-risk individuals [2-6]. Several other large global trials have clearly shown that drug therapies, including metformin [2], acarbose [7] and rosiglitazone [8], can also reduce the incidence of diabetes. Moreover, some of these trials $[2,8]$ also reported a significant increase in the probability of regression of dysglycaemia to normoglycaemia.

All of the completed diabetes prevention trials studied individuals during a period of 3-4 years, after which formal application of the intervention was stopped. However, as diabetes is a lifelong disease, whether the effect of a relatively short exposure to a diabetes prevention intervention is sustained is clearly of interest. If it is sustained, it may lead to a reduction in the development of diabetes-related complications.

The Diabetes Reduction Assessment with Rosiglitazone and Ramipril Medication (DREAM) trial was a multicentre, randomised, placebo-controlled trial of rosiglitazone and/or ramipril in people aged 30 and over with impaired fasting glucose (IFG) and/or IGT [8]. It showed that, during the active treatment phase, rosiglitazone reduced the incidence of the primary composite outcome of diabetes or death by $60 \%$, reduced diabetes alone by $62 \%$ and increased the likelihood of regression to normoglycaemia by $83 \%$. Other analyses also showed that rosiglitazone improved beta cell function [9]. A subset of sites participating in the DREAM trial followed DREAM participants after the active treatment phase of the study was completed. The effect of a median of 3 years of exposure to drug therapy on the incidence of diabetes 1.6 years after therapy had been stopped is reported here.

\section{Methods}

The DREAM trial (clinicaltrials.gov registration number NCT00095654) allocated 5,269 people aged $\geq 30$ years with IFG and/or IGT to either rosiglitazone $8 \mathrm{mg} /$ day or its matching placebo or to ramipril $15 \mathrm{mg} /$ day or its matching placebo. Participants were recruited at 191 centres located in 21 countries between July 2001 and August 2003 and followed for a median period of 3 years on study medication. Detailed descriptions of the study design [10] and final results during the active treatment phase have been published $[8,11]$. All participants provided written informed consent for the active treatment phase. Participants who were taking study medication at their last study visit and were free of diabetes at that time entered a washout phase during which they took single-blind placebo medications. These individuals returned after a median (interquartile range) of 71 (53-86) days for a repeat OGTT.

The DREAM On passive follow-up study was conducted at a subset of 49 clinical sites located in nine countries. All participants provided written informed consent after the DREAM On protocol had been approved by local ethics boards. Consenting participants were invited to have a repeat OGTT 1-2 years after the active study period had ended in addition to measurement of weight, waist and hip circumferences and vital signs, and assessment of oedema. Participants who did not have an OGTT were contacted to ascertain their vital status.

The primary outcome of the DREAM trial was defined as the first occurrence of diabetes or death; death was included to account for the possibility that people who died may have had diabetes that was undiagnosed due to the death. A diagnosis of diabetes was based on confirmed fasting plasma glucose (FPG) or $2 \mathrm{~h}$ plasma glucose (PG) levels $\geq 7.0 \mathrm{mmol} / \mathrm{l}$ or $11.1 \mathrm{mmol} / \mathrm{l}$ respectively during the trial, whereas only one abnormal fasting or $2 \mathrm{~h}$ glucose level was required to diagnose diabetes during the passive follow-up period. During both the trial and the passive follow-up period, diabetes was also defined as a diagnosis by a non-study physician with at least one confirmatory glucose value and prescription of a glucoselowering drug. Regression to normoglycaemia was defined as an $\mathrm{FPG}$ concentration $<6.1 \mathrm{mmol} / 1$ and a $2 \mathrm{~h} \mathrm{PG}<7.8 \mathrm{mmol} / \mathrm{l}$, with both values obtained during a $75 \mathrm{~g}$ OGTT.

Statistical analysis Statistical analyses were restricted to data submitted by the 49 sites that agreed to participate in the follow-up study and that completed a final case report form at the end of the DREAM On passive follow-up period on at least $80 \%$ of site participants who: (1) were on study medication at the end of the DREAM trial, and (2) did not have evidence of diabetes at that visit. All data were analysed according to intention to treat. Participants for whom final diabetes status was not available at the end of the DREAM On study were assumed to be outcome-free at that time. Differences in the baseline characteristics of participants who did and did not complete the follow-up were tabulated and tested using the $t$ test for continuous variables and the $\chi^{2}$ test for categorical variables. Cox proportional hazards models were used to estimate the hazard ratios and $95 \%$ confidence intervals for the effect of allocation to each study drug (stratified by the other) on the primary outcome of diabetes or death and on the secondary outcome of regression to normoglycaemia. Statistical interactions were assessed by the inclusion of an interaction term in the models. 
The effect of allocation to study drugs on the primary and secondary outcomes from the time of randomisation until three different time points was analysed for the included participants: (1) the end of active treatment; (2) the end of the washout phase; and (3) the end of the DREAM On follow-up. An additional analysis was done which was restricted to the period starting at the end of the active treatment period and extending to the end of the washout phase. Sensitivity analyses using the same approach were performed on just those data that were derived from sites that obtained a final glucose tolerance test (in addition to completing a case report form) in $\geq 90 \%$ of participants who were eligible for the DREAM On study. Median changes in fasting and $2 \mathrm{~h}$ PG levels during the DREAM On follow-up study were compared using Wilcoxon rank sum tests. Unadjusted $p$ values were used and a statistical level of significance of 0.05 was used.

\section{Results}

\section{Participation in DREAM On}

As shown in Fig. 1a, 2,464 of the 5,269 DREAM participants (46.8\%) were randomised within research sites that (1) had agreed to participate in the DREAM On extension study and (2) provided final follow-up data regarding primary outcome status in at least $80 \%$ of the randomised participants who did not have diabetes but were taking study medication at the time of the last on-treatment OGTT. Of these, 464 (18.8\%) either died or developed diabetes by the end of the DREAM trial active treatment period, 347 (14.1\%) were ineligible for

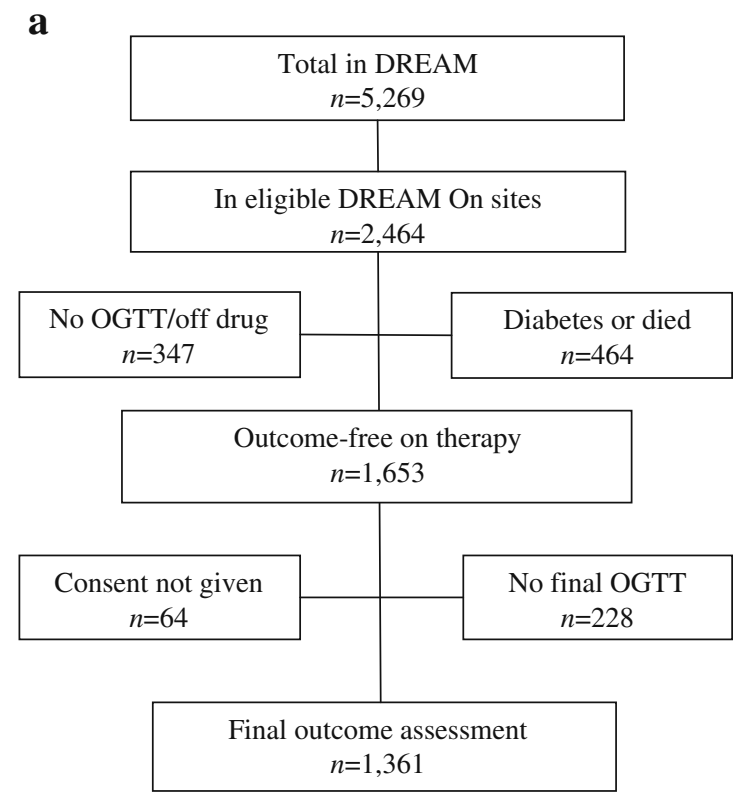

the extension because they were either not on study drug at the last visit or did not have an OGTT, and 1,653 (67.1\%) were alive and had a non-diabetic OGTT result at the last DREAM trial visit while still taking study drug.

Final primary outcome status was available in 1,361 $(82.3 \%)$ of these 1,653 people at the end of DREAM On after a median (interquartile range [IQR]) follow-up period of 581 days $(484,623)$ and 581 days $(490,620)$ in the rosiglitazone and placebo group respectively, and 576 and 586 days in the ramipril and placebo group respectively. The 292 participants for whom final diabetes status was unknown at the end of DREAM On were approximately 2 years older, and had a higher fasting and $2 \mathrm{~h}$ post-load glucose, blood pressure, body mass index, waist circumference and WHR than the 1,361 with known diabetes status (Table 1). The baseline characteristics of the 1,361 with final primary outcomes status who completed the DREAM On follow-up and who had been allocated to rosiglitazone or rosiglitazone placebo are shown in Table 2. At the end of the active treatment period, people who completed DREAM On and who had been allocated to rosiglitazone were heavier, had higher waist and hip circumferences and had more oedema than people allocated to placebo. No differences other than a lower blood pressure were seen for the same 1,361 participants at the end of the active treatment period when analysed according to whether they had been allocated to either ramipril or ramipril placebo.

Long-term effect of exposure to rosiglitazone and/or ramipril on incident diabetes Table 3 displays the effect of the two interventions on the primary outcome of new

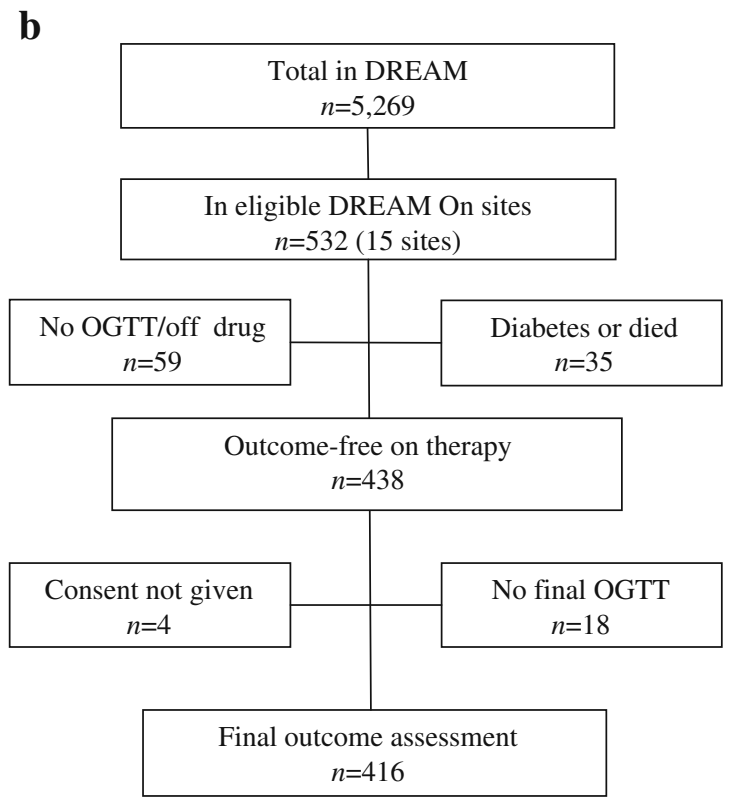

Fig. 1 a Source of participants whose data were analysed for this paper. b Source of participants analysed for the sensitivity analysis, which was restricted to participants from the 15 sites that provided a final outcome assessment for $\geq 90 \%$ of eligible participants 
Table 1 Baseline characteristics of participants who were outcome-free on drug therapy according to whether they completed DREAM On

\begin{tabular}{|c|c|c|c|c|}
\hline Variable & All participants & Final outcome known & Final outcome unknown & $p$ value \\
\hline No. $(\%)$ of participants & $1,653(100)$ & $1,361(100)$ & $292(100)$ & \\
\hline No. $(\%)$ of women & $969(58.62)$ & $789(57.97)$ & $180(61.64)$ & 0.2658 \\
\hline Age (years) & $56.55 \pm 11$ & $56.12 \pm 10.87$ & $58.52 \pm 11.42$ & 0.0007 \\
\hline $\mathrm{FPG}(\mathrm{mmol} / \mathrm{l})^{\mathrm{a}}$ & $5.74 \pm 0.78$ & $5.67 \pm 0.68$ & $6.08 \pm 1.07$ & $<0.0001$ \\
\hline $2 \mathrm{~h} \mathrm{PG}(\mathrm{mmol} / \mathrm{l})^{\mathrm{a}}$ & $7.47 \pm 2.14$ & $7.31 \pm 1.97$ & $8.32 \pm 2.71$ & $<0.0001$ \\
\hline Systolic BP (mm) & $127.39 \pm 16.89$ & $126.56 \pm 16.67$ & $131.38 \pm 17.38$ & $<0.0001$ \\
\hline Diastolic BP (mm) & $77.68 \pm 10.72$ & $77.36 \pm 10.58$ & $79.19 \pm 11.23$ & 0.0096 \\
\hline Heart rate (beats/min) & $72.5 \pm 11.13$ & $72.35 \pm 11.06$ & $73.18 \pm 11.48$ & 0.2595 \\
\hline BMI $\left(\mathrm{kg} / \mathrm{m}^{2}\right)$ & $30.81 \pm 5.71$ & $30.57 \pm 5.72$ & $31.91 \pm 5.58$ & 0.0004 \\
\hline Weight (kg) & $82.57 \pm 17.75$ & $81.89 \pm 17.72$ & $85.8 \pm 17.6$ & 0.0009 \\
\hline Waist circumference, men $(\mathrm{cm})$ & $101.57 \pm 13.99$ & $100.85 \pm 14.21$ & $105.39 \pm 12.14$ & 0.0021 \\
\hline Hip circumference, men $(\mathrm{cm})$ & $106.19 \pm 11.81$ & $105.83 \pm 12$ & $108.1 \pm 10.58$ & 0.0693 \\
\hline WHR, men & $0.96 \pm 0.08$ & $0.95 \pm 0.08$ & $0.98 \pm 0.08$ & 0.0037 \\
\hline Waist circumference, women $(\mathrm{cm})$ & $96.73 \pm 14.57$ & $96.05 \pm 14.1$ & $99.75 \pm 16.22$ & 0.0025 \\
\hline Hip circumference, women $(\mathrm{cm})$ & $111.74 \pm 15.15$ & $111.5 \pm 15.12$ & $112.79 \pm 15.29$ & 0.3102 \\
\hline WHR, women & $0.87 \pm 0.1$ & $0.86 \pm 0.1$ & $0.89 \pm 0.11$ & 0.0088 \\
\hline No. $(\%)$ with peripheral oedema & $165(9.98)$ & $136(9.99)$ & $29(9.93)$ & 1.0000 \\
\hline Serum creatinine $(\mu \mathrm{mol} / 1)$ & $79.6 \pm 28.8$ & $80.1 \pm 30.6$ & $77.3 \pm 17.6$ & 0.1374 \\
\hline No. $(\%)$ receiving rosiglitazone & $888(53.7)$ & $733(53.9)$ & $155(53.1)$ & 0.8462 \\
\hline No. (\%) receiving rosiglitazone placebo & $765(46.3)$ & $628(46.1)$ & $137(46.9)$ & 0.8462 \\
\hline No. $(\%)$ receiving ramipril & $837(50.6)$ & $690(50.7)$ & $147(50.3)$ & 0.9486 \\
\hline No. (\%) receiving ramipril placebo & $816(49.4)$ & $671(49.3)$ & $145(49.7)$ & 0.9486 \\
\hline
\end{tabular}

Data are expressed as mean $\pm \mathrm{SD}$ unless otherwise indicated

${ }^{a}$ These values were those obtained at the washout visit that occurred a median of 71 days after stopping the study drug; all other values were those obtained at the last visit when participants were taking the study drug.

diabetes or death in this subset of 2,464 individuals that occurred from the time of randomisation until the end of: (1) the active treatment period; (2) the 71 day washout period; and (3) the DREAM On follow-up period. Similar to the results originally reported for all randomised participants [8], rosiglitazone reduced the incidence of the primary outcome by $59 \%$ in this subset while it was being taken [hazard ratio (HR) $0.41,95 \%$ CI $0.34-0.50$; $p<0.0001]$. This effect persisted but was attenuated by the end of the washout (HR $0.54 ; 95 \%$ CI $0.46-0.63$; $p<0.0001)$ and by the end of the 1.6 year DREAM On follow-up period (HR $0.61,95 \%$ CI $0.53-0.70 ; p<0.0001$ ), which occurred after a median of 1,572.5 (IQR 1,117, 1,732) days (4.3 years) after randomisation (Fig. 2). When only the 1,653 people who were free of diabetes at the end of the active treatment period were considered, a similar incidence of the primary outcome in people who had been allocated to rosiglitazone $(20.7 \%)$ or placebo $(20.9 \%)$ was noted (HR 1.00; 95\% CI 0.81-1.24; $p=1.0$ ). Similar effects were noted when new diabetes was analysed separately from death; thus, at the end of the 1.6 year DREAM On period, rosiglitazone had reduced the incidence of diabetes by $39 \%$ (HR $0.61,95 \%$ CI $0.53-0.70 ; p<0.0001$ ) with no significant effect on death (HR 0.66; 95\% CI 0.36-1.21). Ramipril did not reduce the primary outcome in this subset of people. There was no evidence of an interaction between rosiglitazone and ramipril with respect to the primary outcome in any of these analyses $(p>0.2)$.

Table 3 also displays the effect of the study drugs on the secondary outcome of regression from IFG or IGT at baseline to the absence of either IFG or IGT (i.e. FPG $<6.1 \mathrm{mmol} / 1$ and $2 \mathrm{~h} \mathrm{PG}<7.8 \mathrm{~mol} / \mathrm{l})$. A total of $52 \%$ of participants on rosiglitazone vs $32 \%$ on placebo experienced regression to normoglycaemia during the active treatment phase of the trial in this subset of 2,464 participants (HR 1.61, 95\% CI 1.42-1.83; $p<0.0001$ ). This effect persisted but was attenuated by the end of the washout period (HR 1.17, 95\% CI 1.02-1.33; $p=0.025$ ) and by the end of the DREAM On follow-up period (HR 1.17. 95\% CI $1.01-1.34 ; p=0.034)$. Because a statistical interaction between rosiglitazone and ramipril $(p=0.03)$ was detected with respect to regression to normoglycaemia at this single time point (i.e. the end of the DREAM On follow-up period), an analysis of the effect of rosiglitazone in the subgroup not taking ramipril was conducted and showed a consistent effect (HR 1.37; 95\% CI 1.11-1.67; 
Table 2 Baseline characteristics of participants who were outcome-free on drug therapy and who completed DREAM On

\begin{tabular}{|c|c|c|c|c|c|c|}
\hline Variable & Rosiglitazone & Placebo & $p$ value & Ramipril & Placebo & $p$ value \\
\hline No. $(\%)$ of participants & $733(100)$ & $628(100)$ & NA & $690(100)$ & $671(100)$ & NA \\
\hline No. $(\%)$ of women & $417(56.9)$ & $372(59.2)$ & 0.4087 & $411(59.6)$ & $378(56.3)$ & 0.2488 \\
\hline Age (years) & $56.2 \pm 10.8$ & $56.0 \pm 11.0$ & 0.6741 & $56.2 \pm 11.0$ & $56.1 \pm 10.8$ & 0.9154 \\
\hline Median (IQR) FPG $(\mathrm{mmol} / \mathrm{l})^{\mathrm{a}}$ & $5.67(5.17,6.11)$ & $5.61(5.20,6.10)$ & 0.5369 & $5.67(5.20,6.11)$ & $5.61(5.22,6.11)$ & 0.7929 \\
\hline Median (IQR) 2 h PG $(\mathrm{mmol} / \mathrm{l})^{\mathrm{a}}$ & $7.00(5.83,8.30)$ & $7.22(5.90,8.70)$ & 0.0524 & $6.94(5.80,8.28)$ & $7.22(5.94,8.72)$ & 0.0140 \\
\hline Systolic BP (mm) & $125.6 \pm 16.2$ & $127.6 \pm 17.1$ & 0.0294 & $124.0 \pm 16.5$ & $129.2 \pm 16.5$ & $<0.0001$ \\
\hline Diastolic BP (mm) & $76.7 \pm 10.6$ & $78.1 \pm 10.5$ & 0.0121 & $76.1 \pm 10.5$ & $78.7 \pm 10.5$ & $<0.0001$ \\
\hline Heart rate (beats/min) & $72.3 \pm 10.9$ & $72.4 \pm 11.2$ & 0.8205 & $72.6 \pm 11.1$ & $72.1 \pm 11.0$ & 0.4659 \\
\hline BMI $\left(\mathrm{kg} / \mathrm{m}^{2}\right)$ & $31.2 \pm 5.7$ & $29.9 \pm 5.7$ & $<0.0001$ & $30.5 \pm 5.7$ & $30.6 \pm 5.7$ & 0.8023 \\
\hline Weight (kg) & $83.8 \pm 17.8$ & $79.7 \pm 17.3$ & $<0.0001$ & $81.9 \pm 17.7$ & $81.9 \pm 17.8$ & 0.9288 \\
\hline Waist circumference, men $(\mathrm{cm})$ & $102.3 \pm 14.2$ & $99.0 \pm 14.0$ & 0.0065 & $100.7 \pm 13.7$ & $101.0 \pm 14.7$ & 0.7892 \\
\hline Hip circumference, men $(\mathrm{cm})$ & $107.7 \pm 12.7$ & $103.6 \pm 10.8$ & 0.0001 & $105.5 \pm 11.6$ & $106.1 \pm 12.4$ & 0.5320 \\
\hline WHR, men & $0.95 \pm 0.07$ & $0.95 \pm 0.08$ & 0.5196 & $0.95 \pm 0.06$ & $0.95 \pm 0.09$ & 0.7937 \\
\hline Waist circumference, women $(\mathrm{cm})$ & $97.1 \pm 14.3$ & $94.9 \pm 13.8$ & 0.0326 & $96.3 \pm 14.2$ & $95.8 \pm 14.0$ & 0.6033 \\
\hline Hip circumference, women $(\mathrm{cm})$ & $113.6 \pm 15.6$ & $109.2 \pm 14.3$ & 0.0001 & $111.4 \pm 15.7$ & $111.6 \pm 14.5$ & 0.8528 \\
\hline Waist/hip ratio, women & $0.86 \pm 0.1$ & $0.87 \pm 0.09$ & 0.0730 & $0.87 \pm 0.11$ & $0.86 \pm 0.08$ & 0.1618 \\
\hline No. $(\%)$ with peripheral oedema & $90(12.3)$ & $46(7.3)$ & 0.0027 & $68(9.9)$ & $68(10.1)$ & 0.9280 \\
\hline Mean serum creatinine $(\mu \mathrm{mol} / \mathrm{l})$ & $78.7 \pm 18.5$ & $81.7 \pm 40.3$ & 0.0685 & $81.2 \pm 38.9$ & $78.9 \pm 18.4$ & 0.1558 \\
\hline
\end{tabular}

Data are expressed as mean $\pm \mathrm{SD}$ unless otherwise indicated

${ }^{a}$ These values were those obtained at the washout visit that occurred a median of 71 days after stopping the study drug; all other values were those obtained at the last visit when participants were taking the study drug

IQR, interquartile range; NA, not applicable

$p=0.003$ ). When only the 1,653 people who were free of diabetes at the end of the active treatment period were considered, a similar incidence of regression to normoglycaemia was noted in people allocated to rosiglitazone or placebo (42.5\% vs $38.2 \%$; HR 1.14; $95 \%$ CI $0.97-1.32$; $p=0.11$ ). Ramipril did not promote regression to normoglycaemia in this subset.

Sensitivity analyses for the effect of rosiglitazone

Two sensitivity analyses were done. First, the analysis was repeated after excluding the 79 rosiglitazone and 46 placebo group participants who took open label thiazolidinediones after the trial had finished. Rosiglitazone's longterm effect on the primary (HR $0.62 ; 95 \%$ CI $0.53-0.70$; $p<0.0001$ ) and secondary (HR 1.22; 95\% CI 1.05-1.41; $p=$ $0.0076)$ outcome remained unchanged. Second, the main analyses assumed that all 292 of the 1,653 (17.7\%) participants for whom primary outcome status was unknown at the end of the DREAM On follow-up were free of the outcome at that time. To explore the effect of missing data on the results, the analyses were repeated for the subset of sites included above $(n=15)$ that provided final diabetes status on at least $90 \%$ of its randomised participants. Figure $1 \mathrm{~b}$ describes the disposition of the 438 participants from these sites. As noted in Table 4, results for this subgroup were concordant with the results for the 1,361 people described above.

Long-term effect of exposure to rosiglitazone and/or ramipril on glucose levels and clinical measures At the time of the DREAM On study visit, the FPG and $2 \mathrm{~h}$ PG levels in participants who had been allocated to rosiglitazone did not differ statistically from the levels in those who had been allocated to placebo (Table 5). Compared with their counterparts on placebo, male participants on rosiglitazone had a greater waist circumference $(p=0.003)$ and WHR $(p=0.002)$ and female participants had a greater hip circumference $(p=0.002)$ and a marginally lower WHR $(p=0.07)$. No significant differences from placebo were noted for the ramipril group.

\section{Discussion}

Diabetes develops when an individual's pancreatic beta cells are unable to produce sufficient insulin to maintain normal glucose homeostasis. An intervention that delays or reduces the incidence of diabetes clearly maintains sufficient insulin production for a longer period of time than one that does not. However, it may accomplish this only while it is being taken and in the process may accelerate or promote 
Table 3 Long-term effect of rosiglitazone and/or ramipril after the DREAM trial at sites that followed at least $80 \%$ of randomised participants

\begin{tabular}{|c|c|c|c|c|c|c|}
\hline & Rosiglitazone & Placebo & $\mathrm{HR}(95 \% \mathrm{CI})$ & Ramipril & Placebo & $\mathrm{HR}(95 \% \mathrm{CI})$ \\
\hline All participants & 2,635 & 2,634 & N/A & 2,623 & 2,646 & NA \\
\hline DREAM On ${ }^{\mathrm{a}}$ & 1,228 & 1,236 & $\mathrm{~N} / \mathrm{A}$ & 1,234 & 1,230 & NA \\
\hline Outcome-free on drug ${ }^{\mathrm{b}}$ & 888 & 765 & N/A & 837 & 816 & NA \\
\hline \multicolumn{7}{|c|}{ Primary outcome of first occurrence of diabetes or death, $n /$ total number $(\%)$} \\
\hline On drug ${ }^{\mathrm{c}}$ & 146/1,228 (11.9) & $318 / 1,236(25.7)$ & $0.41(0.34-0.50)^{\S}$ & 220/1,234 (17.8) & 244/1,230 (19.8) & $0.88(0.73-1.05)$ \\
\hline At end of washout ${ }^{\mathrm{c}}$ & 234/1,228 (19.1) & $385 / 1,236(31.2)$ & $0.54(0.46-0.63)^{\S}$ & $308 / 1,234(25.0)$ & $311 / 1,230(25.3)$ & $0.95(0.81-1.11)$ \\
\hline At end of DREAM On ${ }^{c}$ & $332 / 1,228(27.0)$ & $481 / 1,236(38.9)$ & $0.61(0.53-0.70)^{\S}$ & 407/1,234 (33.0) & $406 / 1,230(33.0)$ & $0.98(0.85-1.12)$ \\
\hline After trial stopped ${ }^{\mathrm{d}, \mathrm{e}}$ & $184 / 888(20.7)$ & $160 / 765(20.9)$ & $1.00(0.81-1.24)$ & $184 / 837(22.0)$ & $160 / 816(19.6)$ & $1.17(0.95-1.45)$ \\
\hline \multicolumn{7}{|c|}{ Secondary outcome of regression to normoglycaemia (FPG $<6.1 \mathrm{mmol} / 1$ and $2 \mathrm{~h} \mathrm{PG}<7.8 \mathrm{mmol} / \mathrm{l}), n /$ total number $(\%)$} \\
\hline On drug ${ }^{\mathrm{c}}$ & $640 / 1,228(52.1)$ & $399 / 1,236(32.3)$ & $1.61(1.42-1.83)^{\S}$ & $544 / 1,234(44.1)$ & $495 / 1,230(40.2)$ & $1.13(1.00-1.28)$ \\
\hline At end of washout ${ }^{\mathrm{c}}$ & $506 / 1,228(41.2)$ & $403 / 1,236(32.6)$ & $1.16(1.02-1.33)^{\ddagger}$ & $477 / 1,234(38.7)$ & $432 / 1,230(35.1)$ & $1.11(0.98-1.27)$ \\
\hline At end of DREAM On ${ }^{\mathrm{c}}$ & $444 / 1,228(36.2)$ & $341 / 1,236(27.6)$ & $1.17(1.01-1.34)^{\dagger}$ & $397 / 1,234(32.2)$ & $388 / 1,230$ (31.5) & $0.98(0.85-1.13)$ \\
\hline After trial stopped ${ }^{\mathrm{d}}$ & $377 / 888(42.5)$ & $292 / 765(38.2)$ & $1.14(0.97-1.32)$ & $334 / 837$ (39.9) & $335 / 816(41.1)$ & $1.01(0.87-1.17)$ \\
\hline
\end{tabular}

NA, not applicable

${ }^{\text {a }}$ People who were originally randomised at participating sites that determined final outcome status in at least $80 \%$ of participants

${ }^{\mathrm{b}}$ People at the participating sites both on study drug and with a non-diabetic OGTT at the last on-treatment visit

${ }^{\mathrm{c}}$ The numerator represents people who developed the outcome from the time of randomisation until the indicated time

${ }^{\mathrm{d}}$ The numerator represents people who were outcome-free at the last on-treatment visit and who developed the primary outcome by the end of the DREAM On follow-up period

${ }^{\mathrm{e}}$ Seventeen of the 344 cases had diagnoses of diabetes ascertained by a non-study physician

${ }^{\dagger} p=0.034 ;{ }^{\ddagger} p=0.025 ;{ }^{\S} p<0.0001$

pancreatic beta cell damage or dysfunction that would be apparent when the intervention is stopped. Alternatively, it may suspend or even reverse a trajectory of pancreatic endocrine dysfunction that would otherwise lead to diabetes.

In this post-treatment analysis of DREAM trial participants with IFG and/or IGT, approximately 3 years of exposure to rosiglitazone reduced the rate of diabetes after approximately $4 \frac{1}{2}$ years by $39 \%$ and promoted regression to normoglycaemia by $17 \%$. We also observed that, during the passive follow-up phase, the incidences of diabetes in

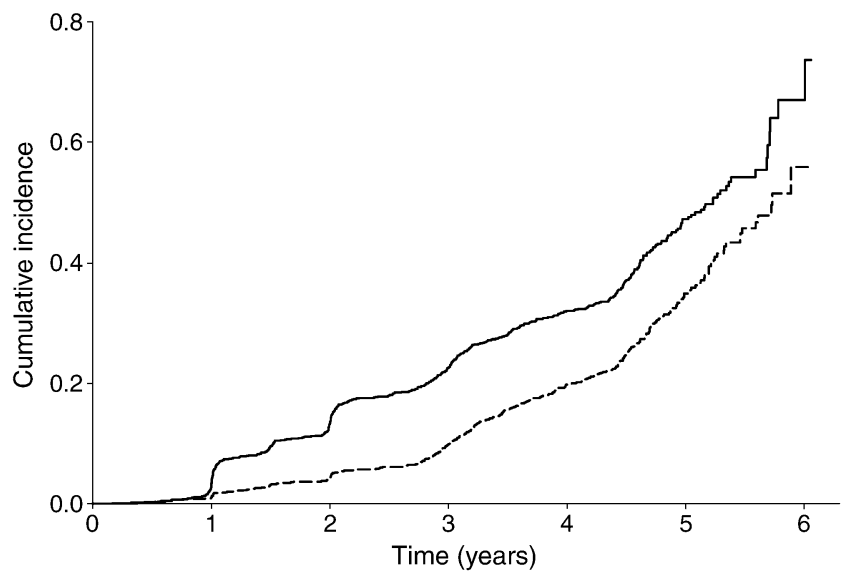

Fig. 2 Incidence of the primary outcome in the rosiglitazone (broken line) and placebo (continuous line) groups during the period from randomisation until the end of the DREAM On follow-up period the rosiglitazone and placebo groups were essentially identical. These findings strongly suggest that rosiglitazone slows the progression of beta cell dysfunction while it is being taken and that the process resumes at the control rate once the drug is stopped. Thus, exposure to $8 \mathrm{mg}$ of rosiglitazone daily neither accelerates nor reverses the processes that lead to dysglycaemia. However, these findings indicate that exposure to rosiglitazone in people with IFG and/or IGT did not simply mask underlying diabetes but rather modified the underlying pathophysiology and as a consequence had a long-term effect on the development of diabetes. With respect to ramipril, the DREAM trial found that ramipril modestly promoted regression to normoglycaemia, but this effect was not apparent within the DREAM On study.

These passive follow-up results with rosiglitazone are similar to those reported with troglitazone (a related TZD) in the American Diabetes Prevention Program (DPP) [12]. In this trial, a mean of 9 months of exposure to troglitazone vs placebo $(n=1,067)$ reduced the rate of diabetes by $75 \%$ during the active treatment period and by approximately $17 \%$ after 4 years from the time of randomisation, with similar rates of diabetes in both groups during the 3 year passive follow-up phase alone. These results are consistent with those from a much smaller study of 266 women with a history of gestational diabetes [13]. A median 30 months' exposure to troglitazone vs placebo reduced the rate of 
Table 4 Long-term effect of rosiglitazone and/or ramipril after the DREAM trial at sites that followed at least $90 \%$ of randomised participants

\begin{tabular}{|c|c|c|c|c|c|c|}
\hline & Rosiglitazone & Placebo & $\mathrm{HR}(95 \% \mathrm{CI})$ & Ramipril & Placebo & $\mathrm{HR}(95 \% \mathrm{CI})$ \\
\hline All participants & 2,635 & 2,634 & $\mathrm{~N} / \mathrm{A}$ & 2,623 & 2,646 & NA \\
\hline DREAM On ${ }^{\mathrm{a}}$ & 275 & 257 & $\mathrm{~N} / \mathrm{A}$ & 270 & 262 & NA \\
\hline Outcome-free on drug ${ }^{\mathrm{b}}$ & 226 & 212 & NA & 229 & 209 & NA \\
\hline \multicolumn{7}{|c|}{ Primary outcome of first occurrence of diabetes or death, $n /$ total number $(\%)$} \\
\hline On drug $^{c}$ & $12 / 275(4.4)$ & $23 / 257(9.0)$ & $0.48(0.24,0.96)^{*}$ & $16 / 270(5.9)$ & $19 / 262(7.3)$ & $0.83(0.43,1.61)$ \\
\hline At end of washout ${ }^{\mathrm{c}}$ & $20 / 275(7.3)$ & $33 / 257(12.8)$ & $0.55(0.31,0.95)^{\S}$ & $27 / 270(10.0)$ & $26 / 262(9.9)$ & $1.02(0.60,1.75)$ \\
\hline At end of DREAM On ${ }^{c}$ & $47 / 275(17.1)$ & $59 / 257(23.0)$ & $0.72(0.49,1.06)$ & $55 / 270(20.4)$ & $51 / 262(19.5)$ & $0.99(0.68,1.46)$ \\
\hline After trial stopped ${ }^{\mathrm{d}}$ & $35 / 226(15.5)$ & $36 / 212(17.0)$ & $0.87(0.54,1.39)$ & $39 / 229(17.0)$ & $32 / 209(15.3)$ & $1.24(0.77,1.99)$ \\
\hline \multicolumn{7}{|c|}{ Secondary outcome of regression to normoglycaemia (FPG $<6.1 \mathrm{mmol} / 1$ and $2 \mathrm{~h}$ PG $<7.8 \mathrm{mmol} / \mathrm{l}), n /$ total number $(\%)$} \\
\hline On drug ${ }^{\mathrm{c}}$ & $162 / 275(58.9)$ & $112 / 257(43.6)$ & $1.49(1.17,1.90)^{\oplus}$ & $156 / 270(57.8)$ & $118 / 262(45.0)$ & $1.49(1.17,1.90)^{\natural}$ \\
\hline At end of washout ${ }^{\mathrm{c}}$ & $143 / 275(52.0)$ & $111 / 257(43.2)$ & $1.29(1.01,1.66)^{\dagger}$ & $136 / 270(50.4)$ & $118 / 262(45.0)$ & $1.22(0.95,1.57)$ \\
\hline At end of DREAM On ${ }^{c}$ & $121 / 275(44.0)$ & $94 / 257(36.6)$ & $1.22(0.93,1.59)$ & $111 / 270(41.1)$ & $104 / 262(39.7)$ & $1.01(0.77,1.33)$ \\
\hline After trial stopped ${ }^{\mathrm{d}}$ & $108 / 226(47.8)$ & $86 / 212(40.6)$ & $1.09(0.82,1.46)$ & $102 / 229(44.5)$ & $92 / 209(44.0)$ & $1.14(0.85,1.52)$ \\
\hline
\end{tabular}

NA, not applicable

${ }^{\text {a }}$ People who were originally randomised at participating sites that determined final outcome status in at least $90 \%$ of its participants;

${ }^{\mathrm{b}}$ People at the participating sites both on study drug and with a non-diabetic OGTT at the last on-treatment visit

${ }^{\mathrm{c}}$ The numerator represents people who developed the outcome between the time of randomisation and the indicated time

${ }^{\mathrm{d}}$ The numerator represents people who were outcome-free at the last on-treatment visit and who developed the outcome by the end of the DREAM On follow-up period

${ }^{\dagger} p=0.044 ;{ }^{\ddagger} p=0.037 ;{ }^{\S} p=0.033 ;{ }^{\circledR} p=0.001$

diabetes by $55 \%$ during the active treatment period. During a median 8 months of passive follow-up, there were fewer cases of new diabetes in the group who were on troglitazone (6 vs 1), but the HR of 0.13 was not significant.

Three studies have reported the long-term postintervention effect of diabetes prevention therapies with other approaches on diabetes incidence. In the Finnish Diabetes Prevention Study, people with IGT who had been randomly allocated to an intensive lifestyle intervention vs standard care for 4 years had a $43 \%$ reduction in diabetes incidence a median of 3 years after the intervention period was stopped [14]. In the DPP, people with IGT were randomly allocated to a lifestyle intervention, metformin or placebo for

Table 5 Final characteristics of participants who were outcome-free on drug therapy and who completed DREAM On

\begin{tabular}{|c|c|c|c|c|c|c|}
\hline & Rosiglitazone & Placebo & $p$ value & Ramipril & Placebo & $p$ value \\
\hline No. $(\%)$ of participants & $733(100)$ & $628(100)$ & NA & $690(100)$ & $671(100)$ & NA \\
\hline Median (IQR) FPG (mmol/l) & $5.70(5.24,6.30)$ & $5.74(5.20,6.30)$ & 0.9438 & $5.70(5.20,6.30)$ & $5.74(5.24,6.30)$ & 0.5627 \\
\hline Median (IQR) 2 h PG (mmol/l) & $7.51(6.07,9.30)$ & $7.69(6.18,9.53)$ & 0.4091 & $7.55(6.10,9.20)$ & $7.60(6.13,9.59)$ & 0.5454 \\
\hline Systolic BP (mm) & $131.3 \pm 16.7$ & $130.6 \pm 16.0$ & 0.4134 & $131.2 \pm 16.3$ & $130.7 \pm 16.5$ & 0.5579 \\
\hline Diastolic BP (mm) & $81.1 \pm 10.1$ & $80.1 \pm 10.0$ & 0.0655 & $80.6 \pm 10.0$ & $80.6 \pm 10.1$ & 0.8943 \\
\hline Heart rate (beats/min) & $72.6 \pm 10.4$ & $71.9 \pm 11.2$ & 0.2724 & $72.3 \pm 10.4$ & $72.2 \pm 11.1$ & 0.9779 \\
\hline BMI $\left(\mathrm{kg} / \mathrm{m}^{2}\right)$ & $31.0 \pm 5.5$ & $30.0 \pm 5.5$ & 0.0017 & $30.4 \pm 5.5$ & $30.6 \pm 5.6$ & 0.6347 \\
\hline Weight (kg) & $82.9 \pm 17.4$ & $79.7 \pm 16.9$ & 0.0010 & $81.4 \pm 17.1$ & $81.4 \pm 17.4$ & 0.9824 \\
\hline Waist circumference, men $(\mathrm{cm})$ & $104.3 \pm 13.2$ & $101.0 \pm 11.8$ & 0.0031 & $103.0 \pm 13.2$ & $102.7 \pm 12.2$ & 0.8237 \\
\hline Hip circumference, men $(\mathrm{cm})$ & $106.4 \pm 11.5$ & $105.3 \pm 12.1$ & 0.2757 & $105.9 \pm 12.0$ & $105.9 \pm 11.6$ & 0.9737 \\
\hline WHR, men & $0.98 \pm 0.07$ & $0.96 \pm 0.07$ & 0.0020 & $0.97 \pm 0.07$ & $0.97 \pm 0.07$ & 0.8429 \\
\hline Waist circumference, women $(\mathrm{cm})$ & $99.5 \pm 13.5$ & $98.0 \pm 13.4$ & 0.1282 & $98.8 \pm 13.6$ & $98.7 \pm 13.4$ & 0.9448 \\
\hline Hip circumference, women $(\mathrm{cm})$ & $112.1 \pm 14.8$ & $108.9 \pm 13.8$ & 0.0017 & $110.7 \pm 14.3$ & $110.5 \pm 14.5$ & 0.8094 \\
\hline WHR, women & $0.89 \pm 0.1$ & $0.90 \pm 0.11$ & 0.0653 & $0.90 \pm 0.10$ & $0.90 \pm 0.11$ & 0.6919 \\
\hline No. (\%) with peripheral oedema & $93(12.7)$ & $65(10.4)$ & 0.2029 & $73(10.6)$ & 85 (12.7) & 0.2371 \\
\hline
\end{tabular}

These values were those obtained at the final DREAM On visit at a median of 581 days after stopping the study drug. NA, not applicable 
3.2 years; subsequently all participants were offered a modified lifestyle intervention and those who had been allocated to metformin were provided with open label metformin. Participants allocated to the lifestyle group achieved a $34 \%$ reduction in diabetes vs placebo over the full 10 year follow-up period and those allocated to metformin (of whom $70 \%$ continued to take metformin) achieved an $18 \%$ reduction [15]. Finally, group allocation of 259 people in China to a 6 year regimen of diet and exercise vs standard care was associated with a $43 \%$ lower incidence of diabetes up to 14 years after the active intervention period finished [16]. These studies clearly highlight the long-term clinical benefits of lifestyle modification for diabetes prevention. However, few individuals in the active treatment group reverted to their pretrial lifestyle, and metformin was continued in the DPP after the trial was unblinded. Conversely, only $6 \%$ of the DREAM On participants who had been allocated to the rosiglitazone arm of the DREAM trial continued to take a thiazolidinedione after the active treatment period. The DREAM findings therefore reflect the effect of a time-limited exposure to rosiglitazone on subsequent diabetes.

A limitation of this analysis is that approximately half of the DREAM sites participated in DREAM On. However, randomisation was stratified within sites so that differences between the rosiglitazone and placebo participants at the non-participating sites would have been similar to those seen at participating sites. Another limitation was the use of only one glucose tolerance test at the final DREAM On visit, and absent data in $17.7 \%$ of the participants at that time. However, the fact that the results were supported by a sensitivity analysis of a subset of participants in whom final outcome status was not available in only $5 \%$ of participants, and were also consistent when anybody taking open label thiazolidinediones after randomisation were excluded, strongly supports the robustness of these findings.

Strong interest in therapies that delay diabetes is based on the inference that delaying diabetes may also delay the onset of diabetes-related serious health consequences. This hypothesis remains unproven for any diabetes prevention therapy. Interest in therapies that delay or prevent diabetes is also based on the inference that delaying diabetes may slow the decline in beta cell function. These DREAM On findings indicate that 3 years of treatment have a legacy effect on incident diabetes, and a recent study in newly diagnosed people with diabetes, in which rosiglitazone therapy achieved durable glycaemic control compared with either metformin or glybenclamide [17], suggests that rosiglitazone preserves beta cell function while it is being taken without harming the beta cell.
Acknowledgements This study was funded by sanofi-aventis Canada, King Pharmaceuticals Research and Development and GlaxoSmithKline.

Duality of interest H. C. Gerstein's institution has received research grants from GlaxoSmithKline (the manufacturer of rosiglitazone) and from sanofi-aventis and King (the manufacturers of ramipril) for research studies being led by him. H. C. Gerstein has received honoraria from speaking and consulting from both GlaxoSmithKline and sanofi-aventis. H. C. Gerstein holds the McMaster University Population Health Institute Chair in Diabetes Research which was established with a donation from sanofi-aventis to McMaster University and which is awarded solely based on University review. R. Bergenstal's institution has received consulting fees and research grants from sanofi-aventis; I. MacKinnon has received a grant for a research study from GlaxoSmithKline; B Zinman has received consulting fees from GlaxoSmithKline, and his institution has received research grants from GlaxoSmithKline; and S. Yusuf has received consulting fees from GlaxoSmithKline and sanofi-aventis and his institution has received research grants from these companies

\section{References}

1. International Diabetes Federation (2009) IDF diabetes atlas, 4th edn. IDF, Brussels

2. Knowler WC, Barrett-Connor E, Fowler SE et al (2002) Reduction in the incidence of type 2 diabetes with lifestyle intervention or metformin. N Engl J Med 346:393-403

3. Ramachandran A, Snehalatha C, Mary S, Mukesh B, Bhaskar AD, Vijay V (2006) The Indian Diabetes Prevention Programme shows that lifestyle modification and metformin prevent type 2 diabetes in Asian Indian subjects with impaired glucose tolerance (IDPP1). Diabetologia 49:289-297

4. Tuomilehto J, Lindstrom J, Eriksson JG et al (2001) Prevention of type 2 diabetes mellitus by changes in lifestyle among subjects with impaired glucose tolerance. $\mathrm{N}$ Engl $\mathrm{J}$ Med 344:1343-1350

5. Kosaka K, Noda M, Kuzuya T (2005) Prevention of type 2 diabetes by lifestyle intervention: a Japanese trial in IGT males. Diabetes Res Clin Pract 67:152-162

6. Pan XR, Li GW, Hu YW et al (1997) Effects of diet and exercise in preventing NIDDM in people with impaired glucose tolerance. The Da Qing IGT and Diabetes Study. Diabetes Care 20:537-544

7. Chiasson JL, Josse RG, Gomis R, Hanefeld M, Karasik A, Laakso M (2002) Acarbose for prevention of type 2 diabetes mellitus: the STOP-NIDDM randomised trial. Lancet 359:2072-2077

8. Gerstein HC, Yusuf S, Bosch J et al (2006) Effect of rosiglitazone on the frequency of diabetes in patients with impaired glucose tolerance or impaired fasting glucose: a randomised controlled trial. Lancet 368:1096-1105

9. Hanley AJ, Zinman B, Sheridan P, Yusuf S, Gerstein HC (2010) Effect of rosiglitazone and ramipril on $\beta$-cell function in people with impaired glucose tolerance or impaired fasting glucose: the DREAM trial. Diabetes Care 33:608-613

10. DREAM Trial Investigators (2004) Rationale, design and recruitment characteristics of a large, simple international trial of diabetes prevention: the DREAM trial. Diabetologia 47:1519-1527

11. Bosch J, Yusuf S, Gerstein HC et al (2006) Effect of ramipril on the incidence of diabetes. N Engl J Med 355:1551-1562 
12. Knowler WC, Hamman RF, Edelstein SL et al (2005) Prevention of type 2 diabetes with troglitazone in the Diabetes Prevention Program. Diabetes 54:1150-1156

13. Buchanan TA, Xiang AH, Peters RK et al (2002) Preservation of pancreatic beta-cell function and prevention of type 2 diabetes by pharmacological treatment of insulin resistance in high-risk hispanic women. Diabetes 51:2796-2803

14. Lindstrom J, Ilanne-Parikka P, Peltonen M et al (2006) Sustained reduction in the incidence of type 2 diabetes by lifestyle intervention: follow-up of the Finnish Diabetes Prevention Study. Lancet 368:1673-1679
15. Knowler WC, Fowler SE, Hamman RF et al (2009) 10-year follow-up of diabetes incidence and weight loss in the Diabetes Prevention Program Outcomes Study. Lancet 374:1677-1686

16. Li G, Zhang P, Wang J et al (2008) The long-term effect of lifestyle interventions to prevent diabetes in the China Da Qing Diabetes Prevention Study: a 20-year follow-up study. Lancet 371:1783-1789

17. Kahn SE, Haffner SM, Heise MA et al (2006) Glycemic durability of rosiglitazone, metformin, or glyburide monotherapy. N Engl J Med 355:2427-2443 